\title{
Evaluation of full scatter convolution algorithm performance in the presence of inhomogeneities using a novel method of three-dimensional film dosimetry
}

\author{
A. Zeinali, L. Mahani, N. Kargar* \\ Department of Medical Physics, Faculty of Medicine, Urmia University of Medical Sciences, Urmia, Iran
}

\section{- Original article}

\author{
*Corresponding authors: \\ Niloofar Kargar, M.Sc., \\ E-mail: \\ niloofar.jeddi@yahoo.com
}

Revised: April 2020

Accepted: June 2020

Int. J. Radiat. Res., April 2021; 19(2): 391-399

DOI: 10.29252/ijrr.19.2.391

\begin{abstract}
Background: Inclusion of air-filled cavities in the head and neck treatment fields due to electronic disequilibrium may lead to uncertainties in predicting dose distribution by treatment planning systems (TPS). In this study the Full Scatter Convolution Algorithm (FSC) used in TiGRT treatment planning systems was evaluated using a novel 3D film dosimetry method. Materials and Methods: 9 pieces of EBT2 films were embedded in a rectangular inhomogeneous head and neck phantom. Three approximately small field sizes including sandwiched EBT films which embedded between phantom slabs were exposed with $6 \mathrm{MV}$ X-ray photons. A homemade computer code was developed in MATLAB for the creation of a 3D dose map of irradiated films and calculation of enclosed volumes which were surrounded by isodose lines in films. Then the calculated dose volumes were compared with the same quantities derived from Dose Volume Histogram (DVH) which is available in TPS outputs. Results: Our results showed significant differences between the results of the film dosimetry and DVH values. The maximum difference of calculated and measured values was observed in volumes surrounded by $95 \%$ isodose curves and $3 \times 3 \mathrm{~cm}^{2}$ field size $(P=0.035)$ and the minimum difference was observed in volumes surrounded by $95 \%$ isodose curves and $5 \times 5 \mathrm{~cm}^{2}$ field size $(P=0.047)$. Conclusions: The results of this study demonstrated overestimated results in volumetric dose calculation of the FSC dose calculation algorithm in the presence of inhomogeneities and the $3 \times 3 \mathrm{~cm}^{2}$ field size. Also, these results confirmed that the utilization of an accurate and comprehensive method such as 3D film dosimetry can be useful for TPS verification.
\end{abstract}

Keywords: 3D film dosimetry, DVH, TPS, inhomogeneities.

\section{INTRODUCTION}

In the last two decades, several advanced treatment techniques of radiation therapy, including intensity-modulated radiation therapy (IMRT), volumetric modulated arc therapy (VMAT), stereotactic radiation therapy (SRT), stereotactic body radiotherapy (SBRT), 3D conformal radiotherapy (3DCRT) and dynamic techniques have been developed (1). The main aim of developing these techniques is to maximize the radiation dose to the tumor while minimizing the dose to healthy tissues (2). Along with the development of radiotherapy techniques, the dose delivery calculation systems known as Treatment Planning Systems (TPSs) also were developed and used in all radiotherapy centers (3). Alongside these signs of progress, evaluation of TPS systems' performance for preventing accidental exposure in radiotherapy seems to be important. Recent studies have shown considerable disturbances 
in dose distribution in the presence of inhomogeneities such as radiotherapy of head and neck and thorax (4-7). According to these researches, the presence of inhomogeneities and loss of charged particle equilibrium occurred in tissues near the air-tissue interfaces, which led to remarkable disagreement between calculated and measured dose so the dose calculation algorithms used in TPSs must be evaluated and verified regularly by precise and accurate methods. Different QA methods were introduced by the American Association of Physicists in Medicine (AAPM) such as Task Group report-53 (TG-53) and International Atomic Energy Agency (IAEA) reports such as Technical Reports-430 (TRS-430) ${ }^{(8,9) . ~ A c c o r d i n g ~ t o ~ t h e s e ~}$ reports, checking both the treatment planning systems and the actual beam delivery issues will be necessary. Consequently, it is very important that the delivered dose to irradiated tissues in the presence of inhomogeneities evaluated by accurate experimental methods and compared with dose delivery calculation system outputs $(10$, $11,6,12)$.

Due to the role of DVHs in the evaluation of three-dimensional dose values in Plan, the AAPM and IAEA were recommended for TPS performance. It is concluded that by using DVHs which shows the volumetric dose values calculated by the TPS algorithm, they must be evaluated via an accurate volumetric dose measurement method in the region with inhomogeneities (13). Although different types of dosimetric methods and techniques are used in practice, application of three-dimensional methods for evaluating dose distribution properties in the presence of inhomogeneities will be useful, also according to AAPM and IAEA recommendations for TPS verification, it can be comparable with data derived from DVHs and applicable for plan evaluation (9).

To verify TPS and dose delivery systems, three-dimensional (3D) dosimeters will be valuable tools. This necessarily motivated the researchers to find and utilize the new approach in 3D dosimetry. Recent studies, based on 3D dosimetry like gel dosimetry, showed a great potential of this method in the evaluation of 3D dose distribution. A different type of data readout in the mentioned method, especially optical-CT and magnetic resonance imaging (MRI), is one of these limitations and challenges. One of these challenges is the scattered light originated from radiation-induced polymer micro-particles within the gel which affected the final readouts (14). The sensitivity of the response to oxygen and the necessity for an external container, where dose-readout is lost, is another limitation of 3D gel dosimeter $(3,15)$. Besides the advantages of this method in the demonstration of 3D dose distribution, the existence of several limitations which mentioned before caused some challenges in the comprehensive use of this method in clinical dosimetry.

According to the AAPM protocols, especially the TG53 report, which point to the need to validate treatment planning systems on the one hand and the use of film dosimetry method for this purpose, employing film dosimetry as a repeatable and accurate method, in particular, high spatial resolution EBT films will be useful as a reliable tool for evaluating the performance and validation of treatment planning systems.

In recent years, a two-dimensional and threedimensional gamma index has been used to evaluate film measurement results and computational results by the treatment planning system as acceptable indicators. Since we use this index only to accept or reject areas with similar and different doses or so-called pass rates, the need to use specialized and commercial software adds to the complexity of this and does not provide any information on the manner of three-dimensional dose distribution, furthermore the effect of dimensionality and non -uniformity of the field on the three-dimensional dose distribution cannot be extracted and compared using gamma-index methods. Dose distribution with the powerful tools of this software tried to be a new and innovative method in the evaluation of treatment planning based on the difference in volumetric dose distribution which is performed using MATLAB software.

Kim et al. introduced a new method of quality assurance $(\mathrm{QA})$ for proton clinical beams via film dosimetry (16). The existence of limitations of gel dosimetry and the results of Kim et al. study 
incited us in this study to develop and use a novel method for evaluating the performance of TPS and photon beam dose distribution based on 3D film dosimetry. The main goal of this study is to introduce a novel 3D film dosimetry method for evaluation of a TPS, which is based on a full scatter convolution (FSC) algorithm in the presence of inhomogeneities and small field sizes.

\section{MATERIALS AND METHODS}

\section{A) Phantom}

A rectangular heterogeneous slab phantom $\left(30 \times 30 \times 23 \mathrm{~cm}^{3}\right)$ was designed and manufactured in this study. The phantom consisted of two $5 \times 5 \times 5 \mathrm{~cm}^{3}$ rectangular air cavities for simulation of Paranasal sinuses. These cavities were designed and placed between rectangular Perspex and Resistant Teflon PTFE slabs (figure 1).

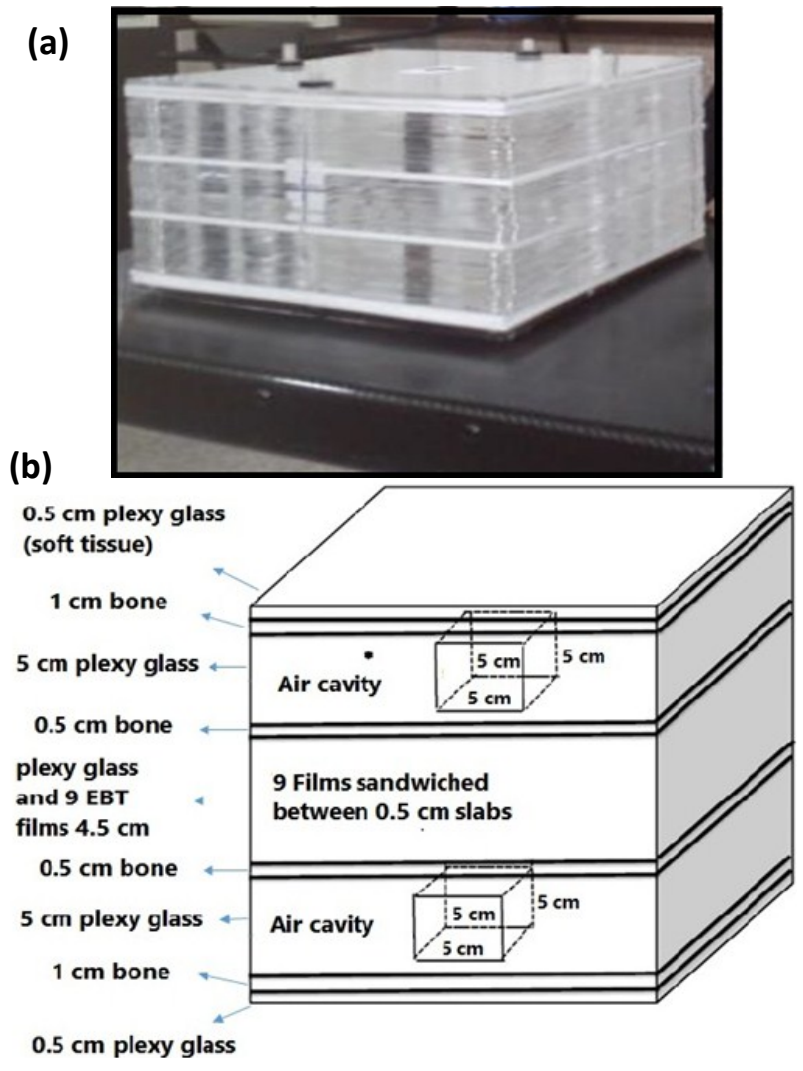

Figure 1. rectangular non- homogenous slab phantom manufactured and used in this study. The photographic image of the phantom (a) and schematic image of the phantom (b).

\section{Film preparation}

9 pieces of GAF chromic films (EBT2 films, International Specialty Products, Inc, Wayne) were used in this study. All of the films were kept in an opaque envelope at room temperature to protect them from visible light. For GAF chromic EBT2 film sharp scissors or guillotine cutters should be used. The cut edge of the film may be stressed and should be avoided for dosimetry analysis. It is important to protect all film fragments from any pressure during film preparation. Latex gloves were used during cutting and marking films to avoid imprinting effects of fingerprints ridges all over the film. The films were prepared on a clean surface area or table (17).

Due to the film's arrangement role in 3D dose readout by computer code and generation of 3D dose distribution, all of the films were numbered before placing in phantom. The size of prepared cut films was considered $1 \mathrm{~cm}$ larger on each side than each field size used for phantom irradiation. For $3 \times 3 \mathrm{~cm}^{2}$ field size with considering $1 \mathrm{~cm}$ margins, cut up to $5 \times 5 \mathrm{~cm}^{2}$ fragments, $4 \times 4 \mathrm{~cm}^{2}$ field size cut up to $6 \times 6 \mathrm{~cm}^{2}$ fragments and $5 \times 5 \mathrm{~cm}^{2}$ field size cut up to $7 \times 7$ $\mathrm{cm}^{2}$ fragments. It should be considered to identify the orientation of the EBT2 films' position, for this purpose the films were marked on their corners. Also, because the EBT2 films' orientation is important in scanning, the sides of 'face-up were noted $(10,12)$.

\section{Irradiation and digitization of films}

A film calibration curve was derived and calculated before exposing phantom to X-ray photons. A dedicated linear accelerator (LINAC- Multi Energy Siemens Primus, Germany) with $10 \times 10 \mathrm{~cm}^{2}$ field size, $100 \mathrm{~cm}$ source to skin (film) distance (SSD) and 6MV photons energy were used $(17,18)$. The films used for Calibration were placed on the central area of a solid water phantom with $20 \mathrm{~cm}$ thickness. Selecting water phantom with $10 \mathrm{~cm}$ thickness under films led to the absorption of all scattered radiations produced by the phantom. To obtain a calibration curve, the fragmented films with $3 \times 3$ $\mathrm{cm}^{2}$ sizes were exposed at dose levels of 0,50 , $100,150,200,250,300,350,400,450,500,550$, 
600, 650, 700 cGy. Three pieces of film sun irradiated and used for measuring background exposure. Then, all films scanned by a dedicated film scanner (Microtek scanner 9800XL, Taiwan) and each film pixel values (PV) were derived.

Due to recommendations by the EBT2 manufacturer, Microtek $9800 \mathrm{XL}$ scanner is widely used as the choice flatbed scanner for EBT2 dosimetry (19). Before beginning the scanning process, the scanner surface was cleaned with sterile gauze and alcohol. One hour before reading films, the scanner was turned on to warm up and stabilize. Because EBT2 film exhibits a different response in portrait orientation which is comparable with the response in landscape orientation, scanning orientation should be kept consistent in all films $(10,17)$. The EBT2 film's fragments were scanned 48 hours after irradiation which allows the films for best post-irradiation coloration.

It is necessary to ensure that all films were consistently scanned with the same face and directed towards the light source of the scanner (20). Images were acquired in 48- bit (red-greenblue (RGB)) color mode with a resolution of 72 dpi and saved with the tagged image file format (TIFF). The red channel information was used for the analysis of results $(10,17,19)$. Then, the film calibration was derived (figure 2). After terminating the calibration process, nine pieces of films were numbered and used for three field sizes. Then all fragments of film sandwiched between two air cavities in the Plexiglas slabs interfaces. Then films were exposed with Parallel-opposed field's technique within rectangular inhomogeneous head and neck phantom. In this study, three field sizes $(3 \times 3$ $\mathrm{cm}^{2}, 4 \times 4 \mathrm{~cm}^{2}$, and $5 \times 5 \mathrm{~cm}^{2}$ ) were examined and the films exposing for every field size were repeated three times.

\section{Treatment planning system}

In this study an FSC based TPS (TiGRTLinatech corporation product, China) was used. For evaluation of TPS performance, the phantom was scanned by a dedicated CT scan machine (Siemens- somatom 2-slices, Germany), images saved in DICOM format and then transferred to the TPS. Then the POP fields were simulated with 600cGy prescription dose (300cGy for each field in each side). The Volume of interest was delineated and DVH plots were obtained (figure 3-5). DVH plots can be utilized as a quantitative tool for evaluation of TiGRT system performance and also will be utilized for comparison with 3D films results in the following steps.

\section{Generation of $3 D$ dose distributions and calculation of enclosed volume}

A homemade computer code was developed which was compatible with MATLAB-Ra 2016 commercial software to produce a 3D dose map of nine exposed films and then by another homemade code, the volume that enclosed isodose lines for each field size was derived and then their results were compared with similar volumetric dose data of TPS (figures 3-5 and tables 1-3). Due to the dimensions of the films used in this study, which were only $1 \mathrm{~cm}$ larger than the field dimensions for each field size, it was not possible to record dose values less than $30 \%$ outside the field, and this was included in the coding. For the doses ranging from $0 \%$ to $30 \%$, the threshold method and interpolation was used in MATLAB code. In this study, isodose $95 \%$ is used as an indicator to compare the results of the treatment planning system and the film dosimetry. The isodose $95 \%$ and the enclosed volume between them were used as an indicator for comparing the results in this study with the ICRU recommendations.

\section{Statistical analysis}

The data obtained from the film dosimetry and treatment planning system was evaluated by the Mann-Whitney non-parametric test using SPSS statistical software. Criterion level of $\mathrm{P}<0.05$ was considered a significant level.

\section{RESULTS}

The film calibration curve was obtained by the code developed in MATLAB software and used in this study as shown in figure 2 . 


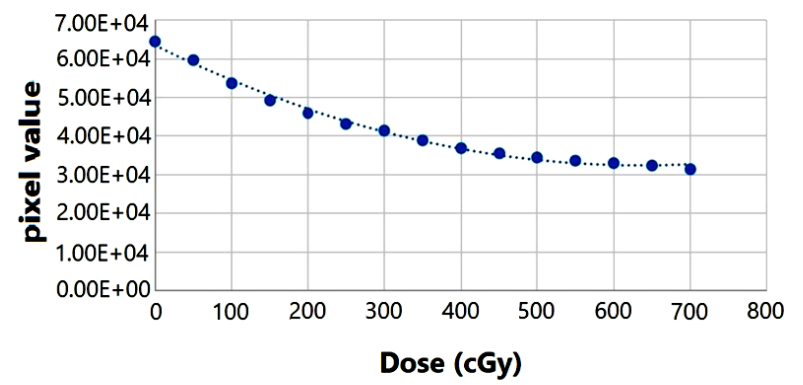

Figure 2. Film calibration curve.

Figures 3-5 show the dose distribution in the 9 films embedded in the phantom for different doses marked in blue to red, for the different field sizes of $3 \times 3,4 \times 4$, and $5 \times 5$ Obtained by MATLAB software. Similar figures were generated for other volumes enclosed with various isodose contours which three of them were selected and showed. In these figures, the $\mathrm{z}$ -axis represents the dose percent in 9 films, the $x$ and $y$ axes represent the dimensions of the field size $(\mathrm{mm})$.

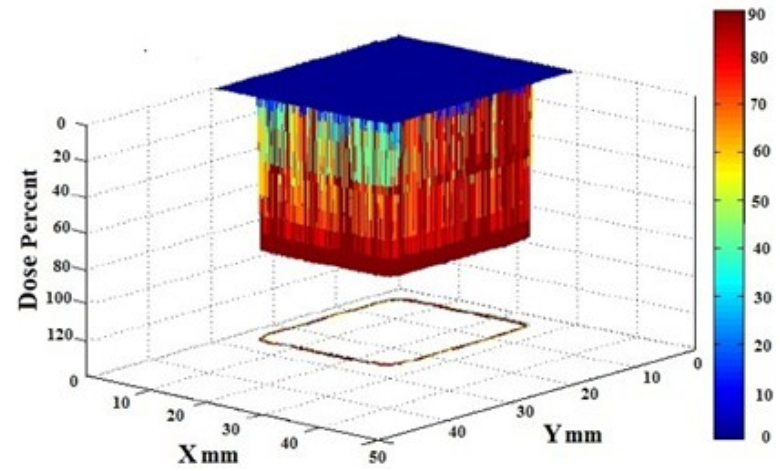

Figure 3. 3D dose distribution for $3 \times 3 \mathrm{~cm}^{2}$ field.

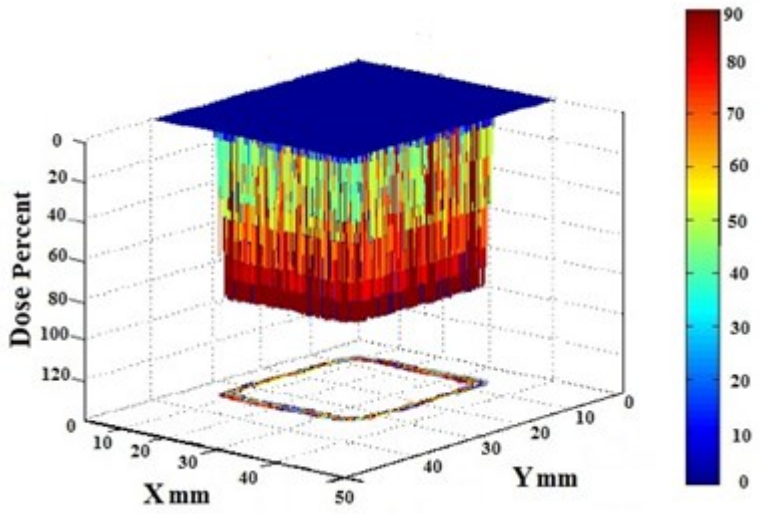

Figure 4. 3D dose distribution for $4 \times 4 \mathrm{~cm}^{2}$ field.

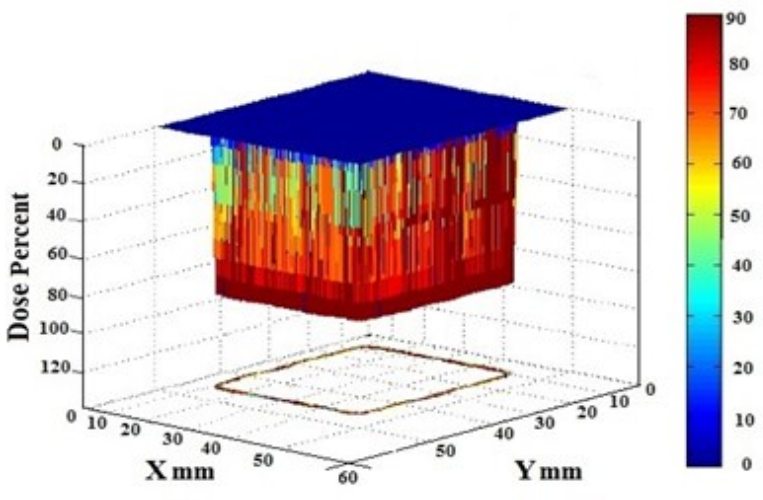

Figure 5. 3D dose distribution for $5 \times 5 \mathrm{~cm}^{2}$ field.

The results of the three trials in the calculation of dose volumes, compared with TPS calculated volumes, are tabulated as below. Tables 1 to 3 shows the volume differences for the $99 \%$ and $95 \%$ doses and lower doses, for the sizes of field $3 \times 3,4 \times 4$, and $5 \times 5$. Based on the results in these tables, there is a significant difference between the values measured by the film dosimetry method and the values calculated by the treatment planning system with $\mathrm{p}<0.05$. These tables also show the results of values including standard deviations (SD) of dose-volume derived from our introduced novel method and DVHs of TPS with some differences. This study showed that as the size of the field decreased, the measured and calculated values of the difference increased significantly, with the difference in the $3 \times 3$ field size reaching about $63 \%$, which necessitates the evaluation of the performance of the TIGRT treatment planning system. It is based on the AAPM protocol.

The use of film dosimetry with highresolution EBT films is recommended as a reliable method in the clinic. According to these tables, for all field sizes between the results of the film dosimetry and the result of the treatment planning system some significant differences were observed. The statistically significant difference for $3 \times 3 \mathrm{~cm}^{2}$ field size obtained $(\mathrm{P}=0.035)$, for $4 \times 4 \mathrm{~cm}^{2}$ obtained $(\mathrm{P}=0.019)$ and for $5 \times 5 \mathrm{~cm}^{2}$ field size obtained $(\mathrm{P}=0.047)$. 
Table 1. Comparison of values obtained from the DVHs with the enclosed volume of isodose that measured by 3D film dosimetry for $3 \times 3 \mathrm{~cm}^{2}$ field size.

\begin{tabular}{|c|c|c|c|}
\hline $\begin{array}{c}\text { \% Dose } \\
\text { (cGy) }\end{array}$ & $\begin{array}{c}\mathbf{V}_{\text {Plan }} \pm S D \\
\text { (cc) }\end{array}$ & $\begin{array}{c}\mathbf{V}_{\text {film }} \pm \text { SD } \\
(\mathbf{c c})\end{array}$ & $\begin{array}{c}\text { \%Difference } \\
\text { plan and film }\end{array}$ \\
\hline$D_{99}=594$ & $7.34 \pm 0.03$ & $4.54 \pm 0.9$ & 61 \\
\hline$D_{95}=570$ & $19.6 \pm 0.03$ & $7.31 \pm 0.68$ & 63 \\
\hline$D_{90}=540$ & $26.89 \pm 0.03$ & $10.7 \pm 0.35$ & 65 \\
\hline$D_{85}=510$ & $29.2 \pm 0.03$ & $16.57 \pm 0.5$ & 48 \\
\hline$D_{80}=480$ & $33.61 \pm 0.03$ & $21.69 \pm 0.7$ & 39 \\
\hline$D_{75}=450$ & $36.7 \pm 0.03$ & $25.42 \pm 0.4$ & 30 \\
\hline$D_{70}=420$ & $36.74 \pm 0.03$ & $28.51 \pm 0.5$ & 22 \\
\hline$D_{65}=390$ & $36.74 \pm 0.03$ & $31.13 \pm 0.4$ & 14 \\
\hline$D_{60}=360$ & $36.74 \pm 0.03$ & $33.12 \pm 0.2$ & 9 \\
\hline$D_{55}=330$ & $36.74 \pm 0.03$ & $35 \pm 0.1$ & 4 \\
\hline$D_{50}=300$ & $36.74 \pm 0.03$ & $36.62 \pm 0.14$ & 0.002 \\
\hline$D_{30}=180$ & $36.74 \pm 0.03$ & $43.35 \pm 1.4$ & 12 \\
\hline
\end{tabular}

Table 2. Comparison of values obtained from the DVHs with the enclosed volume of isodose that measured by 3D film dosimetry for $4 \times 4 \mathrm{~cm}^{2}$ field size.

\begin{tabular}{|c|c|c|c|}
\hline $\begin{array}{c}\% \text { Dose } \\
\text { (cGy) }\end{array}$ & $\begin{array}{c}\mathbf{V}_{\text {Plan }} \pm \text { SD } \\
\text { (cc) }\end{array}$ & $\begin{array}{c}\mathbf{V}_{\text {film }} \pm \text { SD } \\
\text { (cc) }\end{array}$ & $\begin{array}{c}\text { \%Difference } \\
\text { plan and film }\end{array}$ \\
\hline$D_{99}=594$ & $19.6 \pm 0.03$ & $14.36 \pm 1.5$ & 36 \\
\hline$D_{95}=570$ & $36.8 \pm 0.03$ & $21.22 \pm 1$ & 47 \\
\hline$D_{90}=540$ & $56 \pm 0.03$ & $30.9 \pm 1.4$ & 43 \\
\hline$D_{85}=510$ & $63.85 \pm 0.03$ & $40.6 \pm 0.5$ & 47 \\
\hline$D_{80}=480$ & $63.85 \pm 0.03$ & $46.4 \pm 0.8$ & 36 \\
\hline$D_{75}=450$ & $63.85 \pm 0.03$ & $51.51 \pm 0.7$ & 23 \\
\hline$D_{70}=420$ & $63.85 \pm 0.03$ & $55.21 \pm 0.9$ & 14 \\
\hline$D_{65}=390$ & $63.85 \pm 0.03$ & $58.6 \pm 1.2$ & 5 \\
\hline$D_{60}=360$ & $63.85 \pm 0.03$ & $60.65 \pm 1.1$ & 0.002 \\
\hline$D_{55}=330$ & $63.85 \pm 0.03$ & $63.48 \pm 0.25$ & 5 \\
\hline$D_{50}=300$ & $63.85 \pm 0.03$ & $67.40 \pm 0.9$ & 2 \\
\hline$D_{30}=180$ & $63.85 \pm 0.03$ & $76.5 \pm 0.7$ & 13 \\
\hline
\end{tabular}

Table 3. Comparison of values obtained from the DVHs with the enclosed volume of isodose that measured by 3D film dosimetry for $5 \times 5 \mathrm{~cm}^{2}$ field size.

\begin{tabular}{|l|c|c|c|}
\hline $\begin{array}{c}\% \text { Dose } \\
(\mathbf{c G y})\end{array}$ & $\begin{array}{c}\mathbf{V}_{\text {Plan }} \pm S D \\
(\mathbf{c c})\end{array}$ & $\begin{array}{c}\mathbf{V}_{\text {film }} \pm \mathbf{I D} \\
(\mathbf{c c})\end{array}$ & $\begin{array}{c}\% \text { Difference } \\
\text { plan and film }\end{array}$ \\
\hline$D_{99}=594$ & $42.73 \pm 0.03$ & $27.51 \pm 1.2$ & 35 \\
\hline$D_{95}=570$ & $74.68 \pm 0.03$ & $50.72 \pm 1.4$ & 32 \\
\hline$D_{90}=540$ & $82.48 \pm 0.03$ & $61.04 \pm 1.3$ & 27 \\
\hline$D_{85}=510$ & $97.05 \pm 0.03$ & $74.64 \pm 1.5$ & 21 \\
\hline$D_{80}=480$ & $98.40 \pm 0.03$ & $88.09 \pm 1.5$ & 11 \\
\hline$D_{75}=450$ & $103.30 \pm 0.03$ & $93.83 \pm 1.11$ & 10 \\
\hline$D_{70}=420$ & $103.44 \pm 0.03$ & $96.44 \pm 1.04$ & 7 \\
\hline$D_{65}=390$ & $103.83 \pm 0.03$ & $100.5 \pm 1.3$ & 3 \\
\hline$D_{60}=360$ & $104.07 \pm 0.03$ & $102.82 \pm 1.4$ & 1 \\
\hline$D_{55}=330$ & $104.07 \pm 0.03$ & $106.84 \pm 0.7$ & 1 \\
\hline$D_{50}=300$ & $104.07 \pm 0.03$ & $110.09 \pm 0.5$ & 5 \\
\hline$D_{30}=180$ & $104.07 \pm 0.03$ & $112.16 \pm 0.7$ & 7 \\
\hline
\end{tabular}

Table 4. Comparison of the difference between film and plan values at $95 \%$ isodose for $3 \times 3,4 \times 4$ and $5 \times 5 \mathrm{~cm} 2$ field sizes.

\begin{tabular}{|c|c|c|}
\hline field size $\left(\mathbf{c m}^{\mathbf{2}}\right)$ & \%Difference plan and film & P-Value \\
\hline $3 \times 3$ & 63 & 0.035 \\
\hline $4 \times 4$ & 47 & 0.019 \\
\hline $5 \times 5$ & 32 & 0.047 \\
\hline
\end{tabular}

\section{DISCUSSION}

With the improvement of radiotherapy techniques such as 3D-CRT and IMRT during the last decade, the role of treatment planning systems (TPS) in radiation therapy became more prominent. Along with developments in 3D-TPSs, assuring that the treatment planning process is being performed correctly is very important so the application of QA programs for verification of TPS process and plan parameters is thus an important responsibility of the radiation oncology physicist $(8,3,19)$. Although the QA programs will vary widely among different clinics, use of AAPM reports such as TG-53 should allow each clinic to concentrate its QA efforts on those areas which are more important, so the radiation oncology physicist must create an appropriate quality assurance (QA) program for the treatment planning systems and other processes used in their clinics $(8,21,9,22)$.

In this study we introduced a novel method for evaluating TPS based on FSC algorithm (TiGRT) performance in the presence of inhomogeneities and approximate small field sizes. Because of the comprehensive application of this TPS in the field of 3D CRT and IMRT in radiotherapy departments, ensuring of TPS correct performance of preventing accidental exposure in patients will be important. For this purpose, a special rectangular slab phantom composed of different layers for simulation of heterogeneities like head and neck radiotherapy was manufactured and used. Then, the film calibration curves for radio chromic EBT2 films were derived. Thereafter, a homemade computer code was developed in MATLAB for the reconstruction of a $3 \mathrm{D}$ dose map of irradiated films and calculation of enclosed volume to isodose lines obtained.

Finally, equal dose volumes derived by our

Int. J. Radiat. Res., Vol. 19 No. 1, April 2021 
method which was comparable with similar dose-volume data of DVHs. Our results showed a significant difference between calculated and measured values. The maximum difference of calculated and measured values observed for a volume enclosed or surrounded in 95\% isodose curves and $3 \times 3 \mathrm{~cm}^{2}$ field size (\%Difference = $63 \%, \mathrm{P}=0.035$ ) and the minimum difference was observed for a volume enclosed or surrounded in $95 \%$ isodose curves and $5 \times 5 \mathrm{~cm}^{2}$ field size (\% Difference $=32 \%, \mathrm{P}=0.047$ ). For analyzing IMRT and 3D-CRT plans, EBT2 films were shown to produce acceptable agreements between calculated and measured 2D dose distributions $(1,3)$. However, to reduce the occurrence of errors related to the application of radiotherapy technique in IMRT, 3DCRT and other modalities via dosimetric issues, the development and introduction of an accurate practical 3D dosimetry technique may be useful $(23,3)$.

The research which was conducted in 2012 by Kim et al. proved that 3D film dosimetry is a trustworthy method for evaluation of 3D dose distribution in the field of radiotherapy and is one of the authentic procedures for QA of TPS (16). In this study, a 3D film dosimetry method developed and used for evaluation of the 3DCRT dose delivery process and assessing of DVHs accuracy in the TiGRT software. For this purpose, a rectangular head and neck phantom manufactured and radiochromic EBT2 films in conjunction with a Microtek 9800XL scanner in transmission mode were used. This compartment uses in routine radiotherapy QA testing $(19,24)$.

The Results of this study in agreement with other reports, clearly indicated that the presence of inhomogeneities caused significantly comprehensive changes in the interest volumes before and after heterogeneous regions $(4,6,7)$. Also, our results are in agreement with the study of Mesbahi et al., which established research for evaluation of TiGRT performance by using gel dosimetry and Monte Carlo (5). The results of this study presented large discrepancies between the FSC (TiGRT) calculated dose volume and measured values by our method. According to these results, in the presence of heterogeneity and for approximately small fields, the FSC (TiGRT) calculated dose distribution is not in agreement with 3D film dosimetry measurements. For heterogeneous area and approximate small fields, like $3 \times 3 \mathrm{~cm}^{2}$ field size the measured dose distributions and the calculated one were not quantitatively in good agreement. Several factors might explain these inaccuracies and errors (3).

One of the several unavoidable errors is small inaccuracies in the location of single or multiple film sheets that are likely to happen, for example one or less than one-millimeter shifts in placing of films between the phantom slabs can change the outcomes (25). In this study, we couldn't report the values of dose-volume enclosed or surrounded by $5 \%$ isodose lines because the majority of failures occur near the edge of fields (3). In this challenging region (under D30\%), the EBT2 dose readout is inaccurate because of edge artifacts and the TPS results of dose calculation likely to be inaccurate due to difficulty in modeling the edge region.

Our results demonstrated a good agreement between calculated and measured results in the regions with a dose under $60 \%$. According to our results and considering other researcher's reports, finally we concluded that model-based algorithms applied in some TPS dose delivery systems acted weakly in the calculation of doses and have an unacceptable performance in the presence of heterogeneities especially with small and approximate small field sizes. Accordingly, researchers and physicists should find and develop the new algorithms with considering physical, mathematical and biological corrections that able to calculate 3D dose distributions rapidly, accurately and comprehensively.

\section{CONCLUSION}

An experimental procedure based on the 3D film dosimetry method was used to evaluate the FSC dose calculation algorithm in inhomogeneous head and neck phantom. According to the gained results, this study concluded that using a new method based on 3D 
film dosimetry with high spatial resolution EBT2 films in conjunction with a rectangular phantom will be able to be used in radiotherapy departments as a reliable QA method along with other methods recommended by international organizations related to radiation therapy standardizations such as AAPM and ICRU. With consideration of our results which approved some uncertainties in dose delivery calculations, especially in heterogeneous environments and small field size $\left(3 \times 3 \mathrm{~cm}^{2}\right)$, utilization of some accurate and comprehensive methods such as 3D film dosimetry can be useful besides other QA methods.

\section{ACKNOWLEDGEMENT}

This study was supported by Urmia Medical Science University and the Omid radiotherapy department.

\section{Conflicts of interest: Declared none.}

\section{REFERENCES}

1. Chang L, Ho SY, Ding HJ, Lee TF, Chen PY (2014) Dependency of EBT2 film calibration curve on postirradiation time. Medical physics, 41: 021726.

2. Butson MJ, Peter K, Cheung T, Metcalfe P (2003) Radiochromic film for medical radiation dosimetry. Materials Science and Engineering: R: Reports, 41: 61-120.

3. Oldham M, Sakhalkar H, Guo P, Adamovics J (2008) An investigation of the accuracy of an IMRT dose distribution using two-and three-dimensional dosimetry techniques. Medical physics, 35: 2072-80.

4. Martens C, Reynaert N, De Wagter C, Nilsson P, Coghe M, Palmans H, et al. (2002) Underdosage of the upper-airway mucosa for small fields as used in intensity-modulated radiation therapy: a comparison between radiochromic film measurements, Monte Carlo simulations, and collapsed cone convolution calculations. Medical physics, 29: 1528-35.

5. Mesbahi A and Dadgar H (2015) Dose calculations accuracy of TiGRT treatment planning system for small IMRT beamlets in heterogeneous lung phantom. Int J Radiat Res, 13: 345-54.

6. Rana S and Pokharel S (2013) Verification of dose calculation algorithms in a multi-layer heterogeneous phantom using films. Gulf J Oncolog, 1: 63-9.
7. Tillikainen $L$, Helminen $H$, Torsti $T$, Siljamäki S, Alakuijala J, Pyyry J, et al. (2008) A 3D pencil-beam-based superposition algorithm for photon dose calculation in heterogeneous media. Physics in Medicine and Biology, 53: 3821.

8. Fraas B, Doppke K, Hunt M (1998) Quality assurance for clinical radiotherapy treatment planning, AAPM radiation therapy committee TG53. Med Phys, 25: 1829.

9. Jamema S, Upreti R, Sharma S, Deshpande D (2008) Commissioning and comprehensive quality assurance of commercial 3D treatment planning system using IAEA Technical Report Series-430. Australasian Physics \& Engineering Sciences in Medicine, 31: 207-15.

10. Aland T, Kairn T, Kenny J (2011) Evaluation of a Gafchromic EBT2 film dosimetry system for radiotherapy quality assurance. Australasian Physical \& Engineering Sciences in Medicine, 34: 251-60.

11. Kan MW, Leung LH, Peter K (2012) Verification and dosimetric impact of Acuros XB algorithm on intensity modulated stereotactic radiotherapy for locally persistent nasopharyngeal carcinoma. Medical physics, 39: 4705-14.

12. Richley L, John A, Coomber H, Fletcher S (2010) Evaluation and optimization of the new EBT2 radiochromic film dosimetry system for patient dose verification in radiotherapy. Physics in medicine and biology, 55: 2601.

13. Olch AJ (2012) Evaluation of the accuracy of 3DVH software estimates of dose to virtual ion chamber and film in composite IMRT QA. Medical Physics, 39: 81-6.

14. Zakariaee SS, Molazadeh M, Takavar A, Shirazi A, Mesbahi A, Zeinali A (2015) Validation of a Prototype Optical Computed Tomography System. Journal of Medical Signals and Sensors, 5: 123.

15. Wong C, Ackerly T, He C, Patterson W, Powell C, Qiao G, et al. (2009) Small field size dose-profile measurements using gel dosimeters, gafchromic films and microthermoluminescent dosimeters. Radiation Measurements, 44: 249-56.

16. Kim J, Yoon M, Kim S, Shin D, Lee SB, Lim YK, et al. (2012) Three-dimensional radiochromic film dosimetry of proton clinical beams using a gafchromic EBT2 film array. Radiation Protection Dosimetry, 151: 272-7.

17. Horsfield, Megan, Gafchromic ${ }^{\circledR}$ EBT2 film dosimetry in an anthropomorphic head and neck phantom measuring Intensity ModulatedRadiation Therapy (IMRT) dose distributions, Master of Science - Research thesis, Department of Engineering Physics, University ofWollongong, 2012. http://ro.uow.edu.au/theses/3721

18. Kan M, Cheung J, Leung L, Lau B, Yu P (2010) The accuracy of dose calculations by anisotropic analytical algorithms for stereotactic radiotherapy in nasopharyngeal carcinoma. Physics in Medicine and Biology, 56: 397.

19. Sim G, Wong J, Ng K (2013) The use of radiochromic EBT2 film for the quality assurance and dosimetric verification of 3D conformal radiotherapy using Microtek ScanMaker 9800XL flatbed scanner. Journal of Applied Clinical Medical Physics, 14.

20. Alnawaf H, Peter K, Butson M (2012) Comparison of Epson scanner quality for radiochromic film evaluation. Journal of Applied Clinical Medical Physics, 13.

Int. J. Radiat. Res., Vol. 19 No. 2, April 2021 
21. Fuss M, Sturtewagen E, De Wagter C, Georg D (2007) Dosimetric characterization of GafChromic EBT film and its implication on film dosimetry quality assurance. Physics in Medicine and Biology, 52: 4211.

22. Niroomand-Rad A, Blackwell CR, Coursey BM, Gall KP, Galvin JM, McLaughlin WL, et al. (1998) Radiochromic film dosimetry: recommendations of AAPM radiation therapy committee task group 55. Medical physics, 25: 2093-115.

23. McCaw TJ, Micka JA, DeWerd LA (2014) Development and characterization of a three-dimensional radiochromic film stack dosimeter for megavoltage photon beam dosimetry. Medical physics, 41: 052104.

24. Toossi MB, Khorshidi F, Ghorbani M, Mohamadian N, Davenport D (2016) Comparison of EBT and EBT3 RadioChromic Film Usage in Parotid Cancer Radiotherapy. Journal of Biomedical Physics \& Engineering, 6: 1 .

25. Sturtewagen E, Fuß M, Paelinck L, De Wagter C, Georg D (2008) Multi-dimensional dosimetric verification of stereotactic radiotherapy for uveal melanoma using radiochromic EBT film. Zeitschrift für Medizinische Physik, 18: 27-36. 
